\title{
A mutational analysis of U12-dependent splice site dinucleotides
}

\author{
ROSEMARY C. DIETRICH, JOHN D. FULLER, and RICHARD A. PADGETT
}

Department of Molecular Genetics, Lerner Research Institute, Cleveland Clinic Foundation, Cleveland, Ohio 44195, USA

\begin{abstract}
Introns spliced by the U12-dependent minor spliceosome are divided into two classes based on their splice site dinucleotides. The /AU-AC/ class accounts for about one-third of U12-dependent introns in humans, while the /GU-AG/ class accounts for the other two-thirds. We have investigated the in vivo and in vitro splicing phenotypes of mutations in these dinucleotide sequences. A $5^{\prime}$ A residue can splice to any $3^{\prime}$ residue, although $\mathrm{C}$ is preferred. A $5^{\prime} \mathrm{G}$ residue can splice to $3^{\prime} \mathrm{G}$ or $\mathrm{U}$ residues with a preference for $G$. Little or no splicing was observed to $3^{\prime} A$ or $C$ residues. A $5^{\prime} \cup$ or $C$ residue is highly deleterious for U12-dependent splicing, although some combinations, notably $5^{\prime} \mathrm{U}$ to $3^{\prime} \mathrm{U}$ produced detectable spliced products. The dependence of $3^{\prime}$ splice site activity on the identity of the $5^{\prime}$ residue provides evidence for communication between the first and last nucleotides of the intron. Most mutants in the second position of the $5^{\prime}$ splice site and the next to last position of the $3^{\prime}$ splice site were defective for splicing. Double mutants of these residues showed no evidence of communication between these nucleotides. Varying the distance between the branch site and the $3^{\prime}$ splice site dinucleotide in the /GU-AG/ class showed that a somewhat larger range of distances was functional than for the /AU-AC/ class. The optimum branch site to $3^{\prime}$ splice site distance of 11-12 nucleotides appears to be the same for both classes.
\end{abstract}

Keywords: pre-mRNA splicing; spliceosomes; minor class introns

\section{INTRODUCTION}

The complex process of splice site selection, spliceosome formation, spliceosomal activation, and, finally, cleavage and ligation of the RNA chain must ultimately occur with single nucleotide precision. To do otherwise would disrupt the translational frame of downstream exons, leading to incorrect amino acid incorporation and premature translational termination. From the sequence of a number of splice site pairs, a heuristic rule known as the GU-AG rule was developed based on the first and last dinucleotides of the introns (see Crick 1979). By this rule, the nucleotide just $5^{\prime}$ of the GU was joined to the nucleotide immediately $3^{\prime}$ of the AG. Several years later, a genetic and possibly biochemical explanation for this rule was articulated from the results of experiments that examined mutations of the first and last intron $G$ residues (Parker and Siliciano 1993). These experiments confirmed previous results that mutation of either $G$ residue led to aberrant splicing. However,

Reprint requests to: Richard A. Padgett, Department of Molecular Genetics, NE-20, Lerner Research Institute, Cleveland Clinic Foundation, 9500 Euclid Ave., Cleveland, OH 44195, USA; e-mail: padgetr@ccf.org.

Article published online ahead of print. Article and publication date are at http://www.rnajournal.org/cgi/doi/10.1261/rna.7206305. they went on to show that certain combinations of mutations at the first and last positions gave rise to correctly spliced products albeit with lower efficiency than in wild type. The best of these noncanonical combinations was AU-AC with AU-AA producing a lower but measurable level of correct splicing. All other pairs appeared to be inactive. These first experiments were carried out in vivo in Saccharomyces cerevisiae (Parker and Siliciano 1993; Chanfreau et al. 1994; Ruis et al. 1994). Subsequent investigations in mammalian in vitro systems largely confirmed the in vivo yeast findings (Deirdre et al. 1995; Tarn 1996). A suggestion was made for a physical association underlying the genetic results in which G-G, A-C, and A-A pairs of nucleotides could adopt similar non-Watson-Crick base-pairing arrangements and thus fit into the active site(s) of the spliceosome (Parker and Siliciano 1993).

Subsequent work has served to both bolster the generality of the genetic results and to undermine the biophysical model originally proposed to explain them. First, a small number of naturally occurring vertebrate introns have been detected that have AU and AC terminal dinucleotides and that splice using the same U2-dependent spliceosomal mechanism as the majority of introns (Dietrich et al. 1997; Wu and Krainer 1997). Interestingly, this unusual sequence arrangement can be stable over 400 million years 
of evolutionary separation (Dietrich et al. 1997). Second, a careful reexamination of mutant splice sites in yeast has raised the possibility that the terminal nucleotides are not base-pairing with each other but rather are interacting with a common component of the spliceosome (Luukkonen and Seraphin 1997). More recently, a complex set of interactions involving the intron termini, U6 snRNA and the splicing factor Prp8 has been proposed to exist at the catalytic core of the spliceosome (Collins and Guthrie 1999).

Given the prior demonstration of the function of /AU$\mathrm{AC} / \mathrm{termini}$ in U2-dependent splicing, the discovery of another class of spliceosomal introns that appeared to use /AU-AC/ termini preferentially suggested that this new class, the U12-dependent introns, relied on a similar mechanism to insure splicing accuracy (Hall and Padgett 1994). Indeed, when mutations of an intron of this class were tested in which one or both terminal nucleotides were changed to $G$ residues, correct splicing was observed when the termini were /GU and AG/ (Dietrich et al. 1997). Surprisingly, database searches revealed the existence of a previously unrecognized class of U12-dependent introns that naturally contained /GU and AG/ terminal dinucleotides (Burge and Sharp 1997; Dietrich et al. 1997). In fact, the majority of human U12-dependent introns are of the /GU-AG/ type (Levine and Durbin 2001).

Further evidence for an interaction between the terminal nucleotides of U12-dependent introns came from the effects of the mutations described in our previous study (Dietrich et al. 1997). When the $5^{\prime} \mathrm{A}$ was changed to $\mathrm{G}$, the predominant $3^{\prime}$ splice site was shifted two nucleotides downstream to an $\mathrm{AU} /$ dinucleotide instead of the normal $\mathrm{AC} /$ dinucleotide. When the $3^{\prime} \mathrm{C}$ was changed to $\mathrm{G}$, splicing shifted back to the normal position using the new AG/ dinucleotide. These results suggest that the identity of the nucleotide at one end of a U12-dependent intron can influence the splicing machinery to select specific nucleotides at the other end of the intron. This conclusion must be tempered, however, by evidence that other combinations appear to be functional in vivo. For example, naturally occurring human U12-dependent introns with /AU-AA/, /AU-AG/, /AU-AU/, /GU-AA/, /GU-AU/, and /GU-GG/ terminal dinucleotides have been found at low frequencies (Levine and Durbin 2001).

To investigate the possible interactions between the terminal nucleotides in a U12-dependent intron, we have made and tested all 16 pairs of nucleotides using the human nucleolar P120 gene intron F. Previous in vivo and in vitro analysis of splicing in this intron demonstrated that distinct U2- and U12-dependent $5^{\prime}$ and $3^{\prime}$ splice sites are active and that splicing by one or the other system correlates with use of particular splice sites (Dietrich et al. 1997, 2001). Thus, in most cases, the spliceosomal system responsible for a particular splicing pattern in a mutant intron can be deduced from the in vivo splicing pattern. The results show that the U12-dependent spliceosome can accommodate a variety of pairs of terminal nucleotides in a productive reaction. There are clear preferences, however, and some combinations are highly disfavored.

While several combinations of first and last nucleotides appear to be functional for U12-dependent splicing, database searches and previous analyses of mutants indicate that the second position $U$ residue of the $5^{\prime}$ splice site consensus and the next to last position A residue of the $3^{\prime}$ splice site are highly conserved and required for activity of the splice sites. In the case of U2-dependent introns, previous investigations of the effects of mutations in the $U$ of the $5^{\prime} / \mathrm{GU}$ and the $\mathrm{A}$ residue of the $3^{\prime} \mathrm{AG} /$ dinucleotides showed that these residues were important for efficient splicing in vivo and in vitro (Reed and Maniatis 1985; Aebi et al. 1986; Ruis et al. 1994; Gaur et al. 2000). Unlike the case of the terminal $\mathrm{G}$ residues, no combinations of $5^{\prime}$ and $3^{\prime}$ mutations of the $\mathrm{U}$ and $\mathrm{A}$ residues restored function (Ruis et al. 1994). Nevertheless, the potential for an A-U base pair interaction obviously exists and similar interactions appear to play a role in splicing of group II autocatalytic introns (Chanfreau and Jacquier 1993). We therefore investigated possible interactions of these nucleotides in the U12-dependent system by comparing the effects of single and double mutations of the penultimate intron nucleotides. The results show that there are strong mechanistic requirements for both the $U$ at position +2 and the $\mathrm{A}$ at position -2 . In addition, double mutants designed to restore a potential base-pairing interaction between these positions failed to restore function.

As part of our investigation of the function of the terminal intron dinucleotides in U12-dependent splicing, we also examined the functional constraints on the branch point to $3^{\prime}$ splice site distance in a /GU-AG/ U12-dependent intron. Our previous results with an /AU-AC/ intron suggested that a relatively narrow distance window of 10 to $25 \mathrm{nt}$ was necessary for accurate splice site selection (Dietrich et al. 2001). However, the distribution of natural distances observed in U12-dependent introns differs for the /AU-AC/ and the /GU-AG/ classes, with a greater spread of distances seen in the /GU-AG/ subclass (Dietrich et al. 2001; Levine and Durbin 2001). We therefore tested the accuracy and efficiency of splicing as a function of branch point to $3^{\prime}$ splice site distance in a /GU-AG/ intron. The results show that both shorter and longer spacings are functional in a /GU-AG/ intron compared to an /AU$\mathrm{AC} /$ intron.

\section{RESULTS}

\section{In vivo splicing pattern of mutants of the terminal intron nucleotides}

To investigate the role of the terminal nucleotides in the selection of splice sites in a U12-dependent intron, the 
wild-type A and the $\mathrm{A} 1 \mathrm{C}, \mathrm{AlG}$, and $\mathrm{AlU}$ mutations at the $5^{\prime}$ splice site of the P120 minigene expression construct described previously were combined with the wild-type $\mathrm{C}$ and the C99A, C99G, and C99U mutations at the $3^{\prime}$ splice site to give the 16 possible combinations. These constructs were transfected into $\mathrm{CHO}$ cells and the pattern of spliced P120 RNA was assayed after $48 \mathrm{~h}$ using the RT-PCR method described previously. An analysis of the spliced products from these mutants revealed a complex mixture of alternative and cryptic splice site activation (data not shown). In particular, some mutants produced products that were probably due to U2-dependent splicing as was seen earlier (Dietrich et al. 1997).

To simplify the pattern of spliced products, a modified P120 intron F was constructed that eliminated many of the cryptic splice sites near the normal splice junctions. The sequences of the original and new P120 intron F $5^{\prime}$ and $3^{\prime}$ splice sites are shown in Figure 1A. The $5^{\prime}$ splice site region was changed by mutating the last three exon nucleotides to uridines in order to reduce the amount of U2-dependent splicing that occurred with particular splice site mutants (Dietrich et al. 1997). The $3^{\prime}$ splice site region was altered to change the branch site to $3^{\prime}$ splice site distance from 10 to $12 \mathrm{nt}$, which we have previously determined to be the optimum distance (Dietrich et al. 2001). In addition, the AG at position 107 was changed to AU to reduce the U2dependent use of this site (Dietrich et al. 1997). Note that this construct retains the cryptic U2-dependent splice sites at positions 13 and 93 that have been characterized previously (Tarn and Steitz 1996; Dietrich et al. 1997). We have previously noted that splicing using these sites is activated by a number of mutations of the U12-dependent splice sites (Kolossova and Padgett 1997; Incorvaia and Padgett 1998).

All 16 possible combinations of first and last nucleotides were constructed in this new background and tested for splicing by transfection into $\mathrm{CHO}$ cells and analysis by RTPCR. An agarose gel analysis of the spliced products is shown in Figure 1B (top), where the unspliced, spliced, and 13/93 cryptic spliced products are visible. Figure 1B (bottom) is a high-resolution polyacrylamide gel analysis using a radiolabeled primer where the precise sizes of the spliced products can be determined. Lane 1 is the original P120 construct that uses the $3^{\prime}$ splice site at position 99, while lane 2 is the new starting mutant construct that uses a $3^{\prime}$ splice site at position 101 . The splice sites used by each of the mutants was determined by sequence analysis of individual bands excised from a high-resolution nondenaturing polyacrylamide gel.

The results of this analysis are generally in line with the observed splice sites of predicted U12-type introns in bioinformatic analysis of the human genome (Levine and Durbin 2001). When the $5^{\prime}$ splice site has the wild-type A at position 1, the effects of mutations in the last nucleotide of the intron are not large. All four $3^{\prime}$ terminal nucleotides can

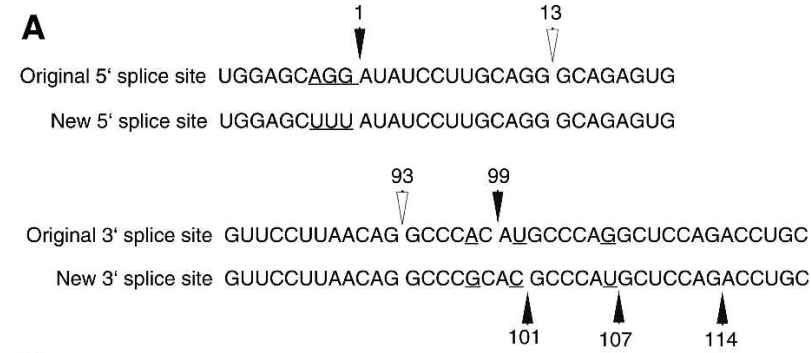

B

5، SS/_U (1) A A 3' SS A_/ (101) \begin{tabular}{ll|l|l|l|l|l|l|l|l|l|l|l|l|l|l|}
\hline & C & C & C & A & G & U & A & G & U & A & G & U & A & G & U \\
\hline
\end{tabular}

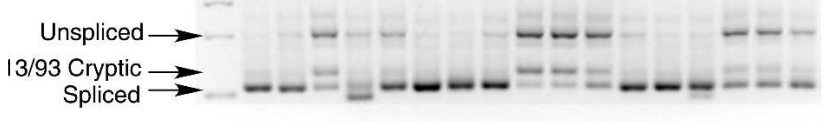

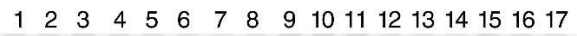

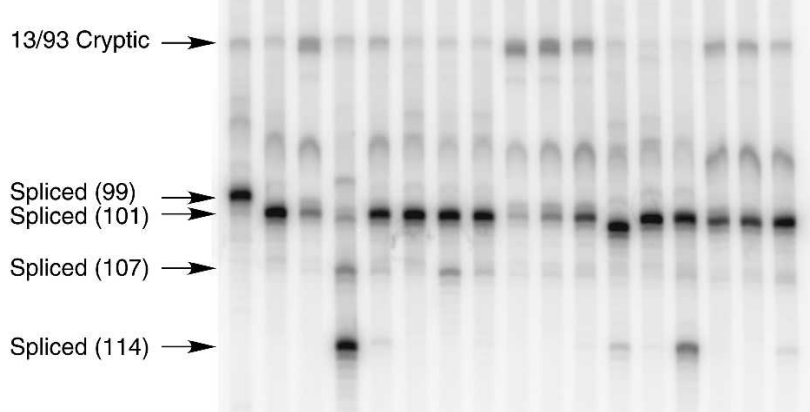

FIGURE 1. (A) Comparison of the $5^{\prime}$ and $3^{\prime}$ splice site regions of human P120 intron F used for the terminal nucleotide mutant analysis. The top lines show the wild-type genomic sequence and the bottom lines show the sequences used as the starting point for the mutants. The changes were designed to reduce the use of nearby cryptic splice sites and to optimize the branch site to $3^{\prime}$ splice site distance. (B) Pattern of in vivo splicing of the original wild type (lane 1 ), the new wild type (lane 2), and the 15 possible mutants of the terminal nucleotides as indicated by the key at the top. The indicated minigene constructs were transfected into $\mathrm{CHO}$ cells and total RNA prepared after $48 \mathrm{~h}$. The splicing pattern of the P120 intron F was determined by RT-PCR amplification and separation of the products on an agarose gel (top) and a denaturing polyacrylamide gel (bottom). The positions of spliced and unspliced PCR products are shown as well as the position of the U2-dependent 13/93 cryptic splice that is activated in some constructs. The numbers at the left indicate the position of the $3^{\prime}$ splice site. In all cases, except for the 13/93 product, the U12-dependent $5^{\prime}$ splice site at position 1 was used.

splice productively to the wild-type /AU $5^{\prime}$ splice site (lanes $2,6,7,8)$. Only the $3^{\prime}$ splice site $G$ mutation activates a significant amount of missplicing in which the AU/ dinucleotide at position 107 downstream of the normal $3^{\prime}$ splice site is activated to a small extent. Thus, all $4 \mathrm{nt}$ can function at the $3^{\prime}$ splice site when the $5^{\prime}$ splice site is an A residue. There is evidence for a preference to use a $\mathrm{C}$ residue, however. Not only is the /AU-AC/ motif the most common in natural introns (Levine and Durbin 2001), but, as can be seen in lane 1 in the original P120 sequence, even when an $\mathrm{AU} /$ dinucleotide is positioned at the favored +12 position relative to the branch site, the $\mathrm{AC} /$ is used exclusively. 
Similarly, a $3^{\prime} \mathrm{G}$ residue appears to be the least active based on its activation of the $107 \mathrm{AU} / 3^{\prime}$ splice site (lane 7).

When the $5^{\prime}$ splice site has a $\mathrm{G}$ at position 1 , the selection of the $3^{\prime}$ splice site becomes more complicated. As described previously (Dietrich et al. 1997), the $5^{\prime}$ splice site A1G mutation leads to the inactivation of the wildtype $\mathrm{AC} / 3^{\prime}$ splice site (lane 4 ). This mutation activates the AG/ 3' splice site at position 114 and, to a lesser extent, the $\mathrm{AU} / 3^{\prime}$ splice site at position 107. As we have shown previously (Dietrich et al. 1997), mutation of the $3^{\prime}$ terminal nucleotide to a $G$ residue restores splicing to the correct 101 site (lane 13). Note that no splicing is detected at the $114 \mathrm{AG} /$ site. When the $3^{\prime}$ terminal nucleotide was mutated to $\mathrm{U}$ (lane 14), significant splicing occurred at the $101 \mathrm{AU} /$ $3^{\prime}$ splice site but the $114 \mathrm{AG} /$ site is also activated. When we tested an A mutation at the 101 site, we also created the sequence AAG. This led predominantly to splicing to the $\mathrm{G}$ nucleotide at position 102 (lane 12). This suggests that the AG/ dinucleotide is much more compatible with a /GU $5^{\prime}$ splice site than is AA/ since an AG/ in a nonconsensus AAG/ motif is used instead of the CAA/ motif. Indeed, when we tested other constructs with /GU-AA/ splice sites, very little splicing to the AA/ $3^{\prime}$ splice site could be observed (data not shown). However, the existence of at least one U12-dependent intron with /GU-AA/ termini (Levine and Durbin 2001) suggests that the AA/ $3^{\prime}$ splice site may function with a /GU $5^{\prime}$ splice site albeit with significantly reduced activity. Thus, the /GU $5^{\prime}$ splice site shows a strong preference for an $\mathrm{AG} / 3^{\prime}$ splice site and a weaker activity with an $\mathrm{AU} / 3^{\prime}$ splice site. $\mathrm{AA} /$ and $\mathrm{AC} / 3^{\prime}$ splice sites are used poorly if at all by the /GU $5^{\prime}$ splice site.

Mutation of the $5^{\prime}$ terminal nucleotide to a $\mathrm{C}$ residue severely inhibited U12-dependent splicing in vivo regardless of the identity of the $3^{\prime}$ terminal residue (lanes 3,9,10,11). Most RNAs either remained unspliced or were spliced using the cryptic 13/93 U2-dependent splice sites. Low levels of apparently correctly spliced RNA could be detected with the /CU-AC/ (lane 3) and /CU-AU/ (lane 11) pairs.

When the $5^{\prime}$ intron nucleotide was mutated to a $\mathrm{U}$ residue, U12-dependent splicing was also inhibited, although not as completely as with the $5^{\prime} \mathrm{C}$ mutant (lanes 5,15, 16,17). All four mutants produced significant amounts of unspliced RNA as well as the 13/93 cryptic product. However, they all also produced roughly similar levels of correctly spliced RNA suggesting that a $U$ at the $5^{\prime}$ splice site can be joined to any nucleotide at the $3^{\prime}$ splice site.

\section{In vivo splicing patterns of mutants of the +2 and $-\mathbf{2}$ intron nucleotides}

In both the U2-dependent and the U12-dependent classes of introns, the $\mathrm{U}$ nucleotide at +2 of the $5^{\prime}$ splice site and the A nucleotide at -2 of the $3^{\prime}$ splice site are virtually $100 \%$ conserved (Burge et al. 1999). The only notable exception is the low frequency occurrence of U2-dependent $5^{\prime}$ splice sites with a $+2 \mathrm{C}$ residue. Most mutations of these positions have been shown to be highly defective for splicing in U2-dependent introns (Reed and Maniatis 1985; Aebi et al. 1986; Ruis et al. 1994; Gaur et al. 2000). We have shown previously that mutation of the $3^{\prime}$ splice site $-2 \mathrm{~A}$ residue in a U12-dependent intron blocks splicing in vivo (Dietrich et al. 1997, 2001). This effect could be due to an essential activity of each of the nucleotides or to an essential interaction between them. One possible interaction could involve a Watson-Crick base pair between the conserved $\mathrm{U}$ at +2 and the A at -2 . While an analysis of an S. cerevisiae U2-type intron failed to uncover a base-pairing interaction between the +2 and -2 positions (Ruis et al. 1994), the critical involvement of many other base-pairing interactions in U12-type intron splicing motivated us to examine this issue in our mammalian U12-type in vivo splicing system.

To examine this potential interaction, we mutated the $\mathrm{U}$ at +2 of the $5^{\prime}$ splice site of the wild-type P120 intron F (see Fig. 1A) to either a $\mathrm{C}$ or an $\mathrm{A}$ residue. We similarly mutated the $A$ at -2 of the $3^{\prime}$ splice site to a $C, U$, or $G$ residue. We also combined each of these mutants to generate five single mutants and six double mutants. Figure 2 shows the RTPCR analysis of the in vivo splicing patterns of these
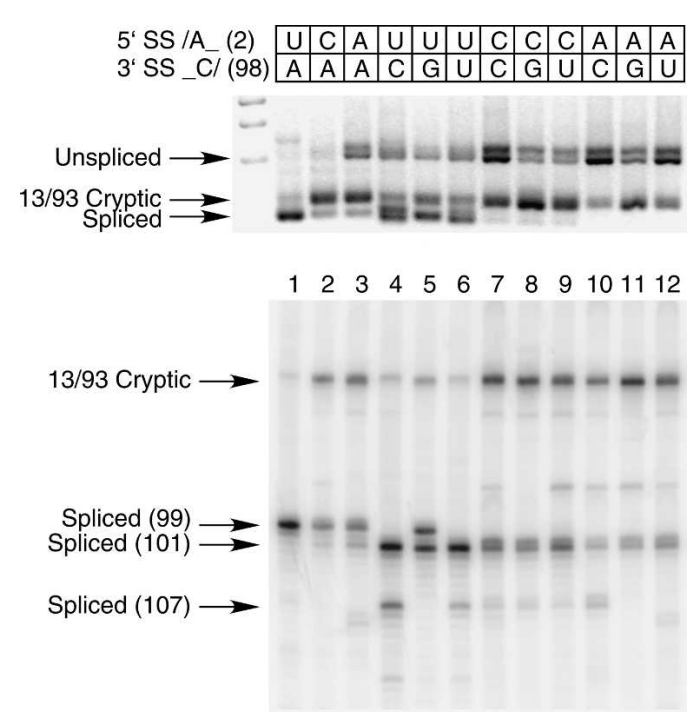

FIGURE 2. In vivo splicing of mutants in the $5^{\prime}$ splice site $+2 \mathrm{U}$ and $3^{\prime}$ splice site $-2 \mathrm{~A}$ positions. The mutants were constructed in the original P120 wild type shown in Figure 1A (top). Correctly spliced RNA uses the $3^{\prime}$ splice site at 99 . Shown are the original wild type (lane 1), two single mutants of the $5^{\prime}$ splice site +2 position (lanes 2,3), three single mutants of the $3^{\prime}$ splice site -2 position (lanes 4-6), and six double mutants (lanes 7-12). The indicated minigene constructs were transfected into $\mathrm{CHO}$ cells and total RNA prepared after $48 \mathrm{~h}$. The splicing pattern of the P120 intron F was determined by RTPCR amplification and separation of the products on an agarose gel shown at the top and a denaturing polyacrylamide gel at the bottom. The positions of spliced and unspliced PCR products are shown as well as the position of the U2-dependent 13/93 cryptic splice that is activated in some constructs. The numbers at the left indicate the position of the $3^{\prime}$ splice site. In all cases, except for the 13/93 product, the U12dependent $5^{\prime}$ splice site at position 1 was used. 
mutants. Lane 1 shows the splicing of the wild-type intron. When the $+2 \mathrm{U}$ was mutated to $\mathrm{C}$ or $\mathrm{A}$, correct splicing to the wild-type $\mathrm{AC} / 3^{\prime}$ splice site was drastically reduced with concomitant activation of the 13/93 cryptic splice sites (lanes 2,3). When the -2 A was mutated to $C, G$, or $U$, overall splicing was inhibited as shown by the accumulation of unspliced RNA (lanes 4-6). However, some splicing using the wild-type U12-dependent $5^{\prime}$ splice site did occur, but in all cases, mainly to the AU/ dinucleotide at position 101 in agreement with our previous results (Dietrich et al. 2001). Some splicing also occurred at the AG/ dinucleotide at position 107. Only in the case of the $-2 \mathrm{G}$ mutation did detectable splicing occur at the normal 99 position. This suggests that a $3^{\prime} \mathrm{GC} /$ dinucleotide can participate in splicing to a limited extent.

In vivo splicing of the double mutants shown in lanes $7-$ 12 was severely inhibited in all cases. The top part of Figure 2 shows that the unspliced and 13/93 cryptic spliced products predominate, with almost no signal in the region of the correctly spliced products. The high-resolution gel in the lower section of Figure 2 shows that a small amount of splicing to the AU/ dinucleotide at position 101 can be detected. Note that none of the double mutant combinations splices better than the $+2 \mathrm{C}$ and A single mutants shown in lanes 2 and 3, suggesting that none of these combinations restores an essential interaction. In particular, note that the $+2 \mathrm{~A}$ and $-2 \mathrm{U}$ combination shown in lane 12 could form a base pair that inverts the putative U-A base pair between the wild-type splice sites. Similarly, the $+2 \mathrm{C}$ and $-2 \mathrm{G}$ combination shown in lane 8 could form a C-G base pair in place of a wild-type U-A base pair. Since none of these combinations restores splicing, we conclude that the essential splicing function of the +2 and -2 nucleotides in the splice site dinucleotides involves other than Watson-Crick base-pairing with each other in the U12-dependent splicing system.

\section{Effects of branch point to $3^{\prime}$ splice site distance in GU-AG introns}

We have previously described the effects on $3^{\prime}$ splice site usage caused by varying the distance between the branch site and the $3^{\prime}$ splice site in the wild-type /AUAC/ P120 intron F (Dietrich et al. 2001). Our results showed that efficient use of a $3^{\prime}$ splice site occurred when the distance to the branch site $A$ residue was between 10 and $20 \mathrm{nt}$, with a pronounced preference for a distance of $12 \mathrm{nt}$. This is similar to the range of distances observed in natural /AU-AC/ U12-dependent introns (see Fig. 1 of Dietrich et al. 2001). We also reported that the range of natural distances in /GU-AG/ introns was greater than that of /AU-AC/ introns. This finding was further supported by an analysis of a larger sample of human U12-dependent introns that showed a broad peak in the distance distribution between 12 and $16 \mathrm{nt}$ for /GU-AG/ introns (Levine and Durbin 2001).

To investigate the mechanistic basis of this difference between the two subclasses of U12-dependent introns, we have examined the effects on in vivo and in vitro splicing of altering the branch site to $3^{\prime}$ splice site distance in a /GUAG/ intron. For this analysis, we used the G-1A/A1G 5' splice site mutant of P120 intron F and combined it with $3^{\prime}$ splice sites based on the C99G mutant to give the mutant labeled GU-AG +10 in Figure 3. The in vivo and in vitro splicing phenotype of this mutant was reported previously (Dietrich et al. 1997). Additional mutants were constructed as shown in Figure 3 to both increase and decrease the distance between the branch site and the $3^{\prime}$ splice site. These mutants were analyzed for their in vivo splicing phenotypes by transfection into $\mathrm{CHO}$ cells followed by RT-PCR amplification of the intron F region of the minigene construct. Their in vitro splicing phenotype was determined by incubating RNA transcripts of the mutants in HeLa cell nuclear extracts under U12-dependent splicing conditions followed by RT-PCR analysis of the spliced

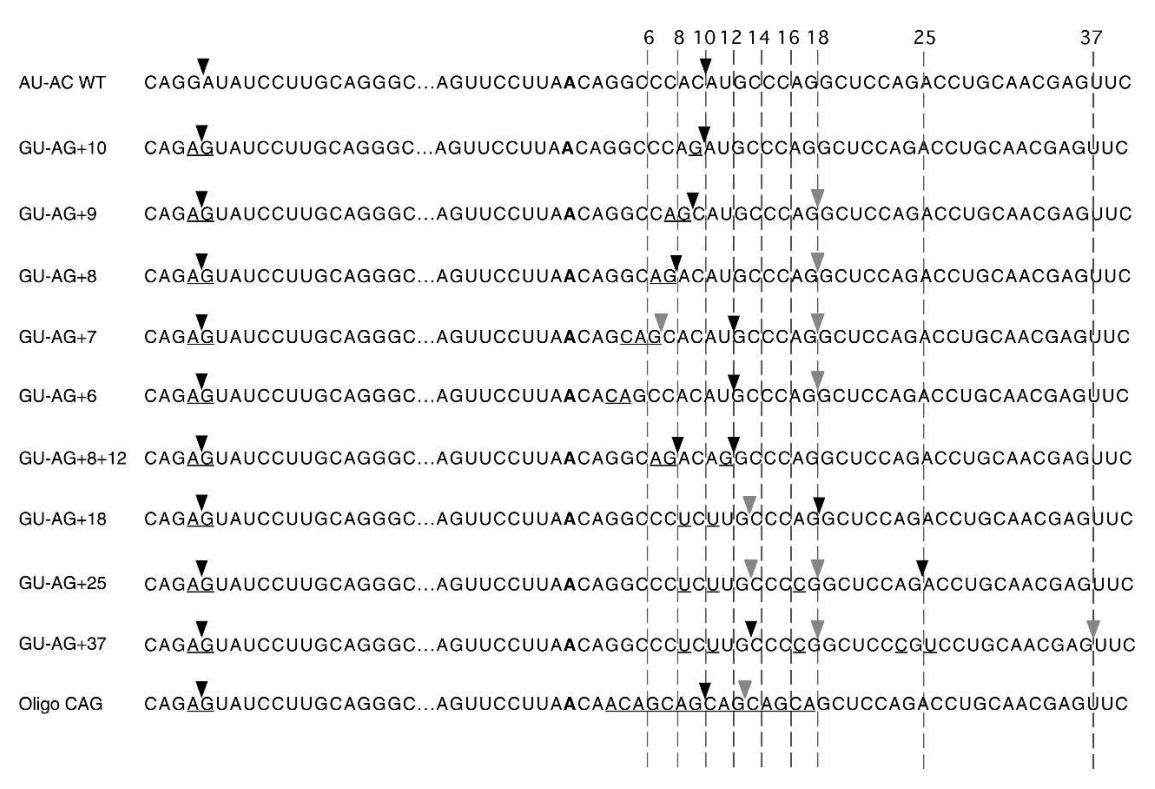

FIGURE 3. Sequences of the $3^{\prime}$ splice site mutants used to determine the branch site to $3^{\prime}$ splice site spacing constraints for a /GU-AG/ U12-dependent intron. The top line is the original P120 intron F sequence with the consensus $5^{\prime}$ splice site and branch site sequences boxed. The second line is the /GU-AG/ version of this intron, which includes a mutation of the $-1 \mathrm{G}$ to an A adjacent to the $5^{\prime}$ splice site. This reduces the use of this $5^{\prime}$ splice site by the U2dependent spliceosome (Dietrich et al. 1997). The $3^{\prime}$ splice sites of the constructs are shown at the right of the figure. The common branch site is shown in bold and the mutated positions are underlined. The sites of in vivo splicing for each construct are indicated by the arrows. Major sites are indicated by dark arrowheads and minor sites are indicated by light arrowheads. The numbers at the top refer to the distance in nucleotides between the branch site adenosine and the dashed lines. 
products as described previously (Dietrich et al. 1997, 2001). The PCR products from both the in vivo and in vitro RNAs were then run together on a high-resolution polyacrylamide gel (Fig. 4). The RNA products of the in vitro reactions are shown in Figure 5.

The wild-type branch site to $3^{\prime}$ splice site distance in $\mathrm{P} 120$ intron $\mathrm{F}$ is $10 \mathrm{nt}$. We first examined the effect of reducing this distance by single nucleotide steps to $6 \mathrm{nt}$. Consistent with the results shown above, when an AG/ dinucleotide is positioned at +10 , all splicing is to this site. As the branch site to $3^{\prime}$ splice site distance is decreased from +10 to +6 , the efficiency of splicing to the upstream $\mathrm{AG} /$ decreases. In the +9 and +8 mutants, the usage of the upstream AG/ was only moderately reduced both in vivo and in vitro, accompanied by modest activation of the +18 $\mathrm{AG} /$ site. In the +7 and +6 mutants, the use of the upstream site is drastically reduced. However, rather than leading to activation of the AG/ dinucleotide at +18 , these mutants caused activation of the AU/ dinucleotide at +12 . The overall amount of splicing in this series of mutants is similar in the in vitro assay as shown in Figure 5. The use of the $+12 \mathrm{AU} / 3^{\prime}$ splice site in the +7 and +6 mutants was also associated with a defect in the second step of splicing in vitro as indicated by the increase in the $5^{\prime}$ exon intermediate band (Fig. 5, lanes 9,11).

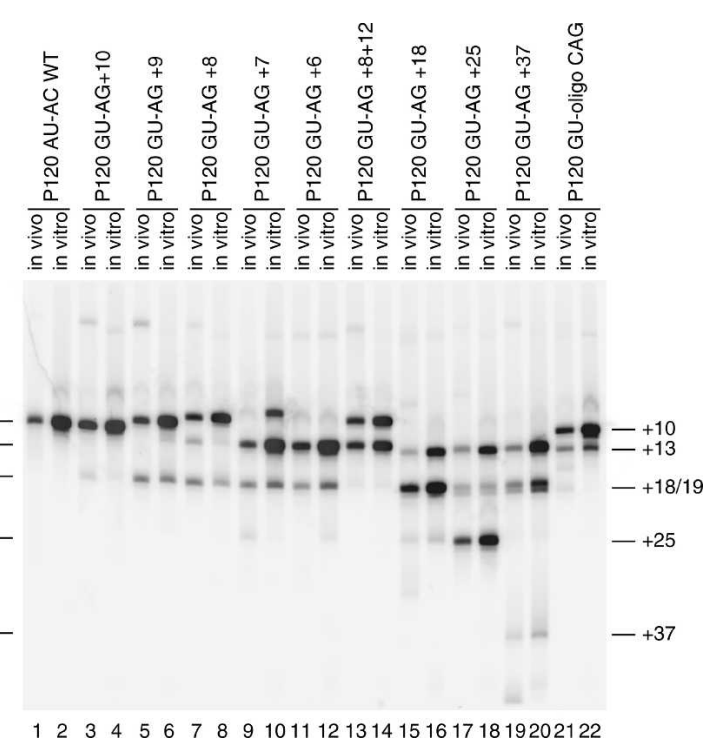

FIGURE 4. Patterns of in vivo and in vitro splicing of $3^{\prime}$ splice site spacing mutants. The indicated minigene constructs were either transfected into $\mathrm{CHO}$ cells and total RNA prepared after $48 \mathrm{~h}$ or transcribed in vitro and subjected to splicing under U12-dependent conditions in HeLa cell nuclear extract (see Fig. 5). The splicing patterns of the P120 intron $\mathrm{F}$ in both the in vivo and in vitro RNA samples were determined by RT-PCR amplification and separation of the products on a denaturing polyacrylamide gel. The numbers at the left indicate the distance in nucleotides between the branch site and the $3^{\prime}$ splice site in each product. The results are summarized in Figure 3 . In all cases, the U12-dependent $5^{\prime}$ splice site at position 1 was used.

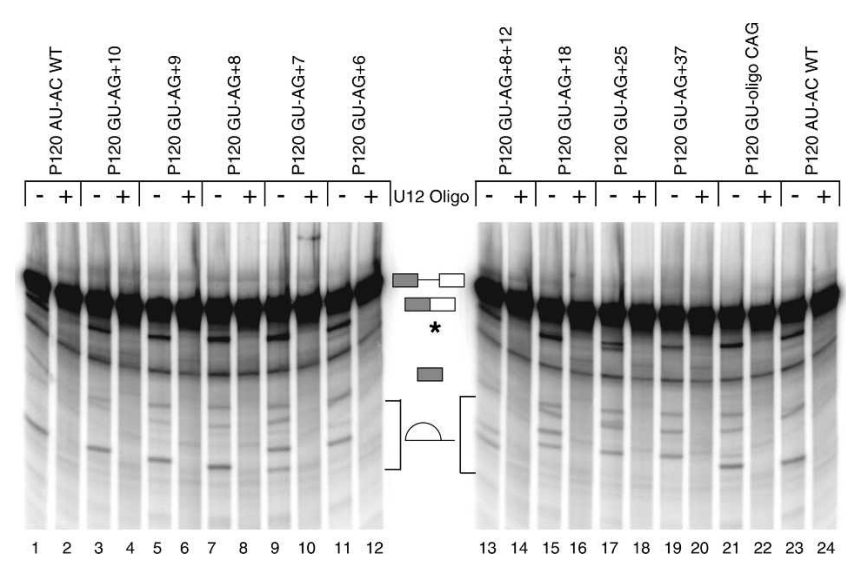

FIGURE 5. In vitro splicing patterns of P120 $3^{\prime}$ splice site spacing mutants. Templates for in vitro transcription of the indicated $3^{\prime}$ splice site constructs were produced by PCR amplification from the indicated minigene constructs. Equal amounts of transcribed precursor RNAs were spliced in vitro. All reactions contained an anti-U2 snRNA $2^{\prime}$-O-methyl oligonucleotide that inhibits U2-dependent splicing. An anti-U12 snRNA $2^{\prime}$-O-methyl oligonucleotide was also added to even numbered lanes to inhibit U12-dependent splicing. The structures of the various RNA products are shown in the middle and correspond, from top to bottom, to the unspliced precursor, spliced exon product, the exon 1 intermediate generated by the first step of splicing, and the excised lariat intron generated by the second step of splicing. The lariat introns from different constructs migrate differently due to the varying length of RNA $3^{\prime}$ of the branch. A degradation product that migrates below the position of spliced exons is labeled with an asterisk.

These results differ from our previous findings with this same intron using /AU-AC/ splice sites (Dietrich et al. 2001). In this study, virtually no correct splicing was observed when the branch site to $3^{\prime}$ splice site distance was reduced from 10 to 9. This led us to suggest that a steric constraint in the U12dependent spliceosome might be preventing use of $3^{\prime}$ splice site dinucleotides that were closer than $10 \mathrm{nt}$ to the branch site. However, the data above suggest that this is not the case. To determine if there is a serious constraint on the use of proximal $3^{\prime}$ splice sites, we constructed a mutant in which $3^{\prime}$ splice site AG/ dinucleotides were positioned 8 and $12 \mathrm{nt}$ away from the branch site. Figure 4, lanes 13 and 14, show that in this mutant, use of the two $3^{\prime}$ splice sites was roughly equal both in vivo and in vitro.

To investigate the distal extreme of functional branch to $3^{\prime}$ splice site distances, mutants were constructed in which the first AG/ dinucleotide was located at distances of 18,25 , and $37 \mathrm{nt}$. Figure 4 shows that the AG/ at a distance of 18 was the predominant site of splicing, but a small amount of splicing also occurred at the UG/ at +13 . A similar result was seen when the distance was increased to $25 \mathrm{nt}$. However, at a distance of $37 \mathrm{nt}$, almost no splicing occurred at the AG/, but instead this mutant led to further activation of the +13 $\mathrm{UG} /$ and the $+18 \mathrm{CG} /$ and $+19 \mathrm{GG} /$. As in the +7 and +6 cases, splicing to these non-AG/ $3^{\prime}$ splice sites in the $+18,+25$, and +37 mutants was associated with increased $5^{\prime}$ exon intermediate in the in vitro reactions shown in Figure 5. 


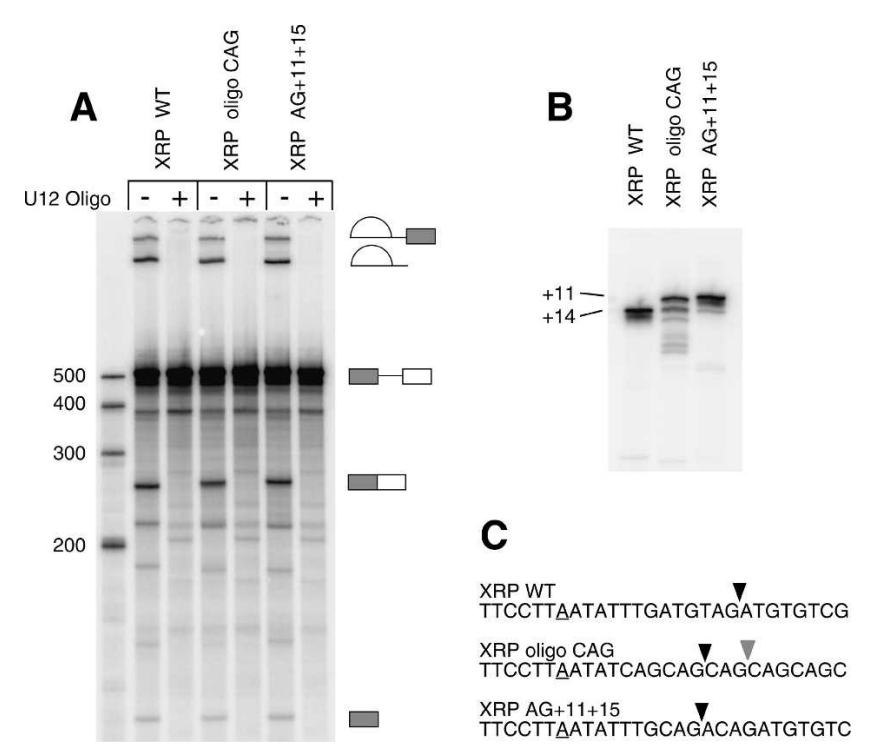

FIGURE 6. In vitro splicing patterns of XRP $3^{\prime}$ splice site spacing mutants. Templates for in vitro transcription were produced by PCR amplification from the XRP construct described by McConnell et al. (2002). Equal amounts of transcribed precursor RNAs were spliced in vitro. All reactions contained an anti-U2 snRNA 2'-O-methyl oligonucleotide that inhibits U2-dependent splicing. An anti-U12 snRNA $2^{\prime}$-O-methyl oligonucleotide was also added to even numbered lanes to inhibit U12-dependent splicing. (A) Products of in vitro splicing reactions run on a denaturing polyacrylamide gel. The bands corresponding to the precursor RNA and the intermediates and products of splicing are diagramed. $(B)$ RT-PCR amplification of the spliced products from the reactions in $A$ were run on a denaturing polyacrylamide gel. (C) The sequences of the branch and $3^{\prime}$ splice site regions of the wild-type and mutant XRP constructs are shown. The major sites of splicing are marked with black arrowheads; minor sites are marked with gray arrowheads.

These results show that, as in the case of the /AU-AC/ class of U12-type introns, the range of functional branch to $3^{\prime}$ splice site distances in the /GU-AG/ class of introns is relatively small. Our previous work suggested the range was from 10 to $\sim 20 \mathrm{nt}$ for the /AU-AC/ class (Dietrich et al. 2001), while the work here suggests that the range is from $\sim 8$ to between 25 and $37 \mathrm{nt}$ for the /GU-AG/ class.

Finally, to determine the optimum branch to $3^{\prime}$ splice site distance, we constructed a mutant with five tandem copies of the consensus $3^{\prime}$ splice site sequence of CAG positioned 7, 10, 13, 16, and 19 nt from the branch site. The splicing phenotype of this mutant, P120 GU-oligo CAG, is shown in Figures 4 and 5, lanes 21 and 22. Both the in vivo and in vitro patterns show that the CAG at a distance of 10 was used preferentially with some splicing to the CAG at a distance of $13 \mathrm{nt}$. The sites at 7,16 , and 19 were not used at a detectable level, suggesting that there is a clear preference for a small range of distances $\sim 10-12$ nt. A similar construct using duplicated CAC sites in the /AU-AC/ version of this intron yielded a preferred distance of $12 \mathrm{nt}$ (Dietrich et al. 2001).

The above results are somewhat different than what is observed in natural /GU-AG/ U12-dependent introns, where the peak of a broad distribution of distances is at $\sim 16 \mathrm{nt}$ (Levine and Durbin 2001). The peak of the distance distribution of /AU-AC/ introns is closer to the optimum $12 \mathrm{nt}$ spacing. It is possible that our strategy of converting a natural /AU-AC/ intron to a /GU-AG/ intron somehow skewed the results of the present study. However, other than the nucleotide immediately preceding the $5^{\prime}$ splice site (the $-1 \mathrm{nt}$ ), there are no significant differences in the consensus sequences for the two classes. In the present study we changed the $-1 \mathrm{nt}$ from a $\mathrm{G}$ to an $\mathrm{A}$ to take this difference into account.

Nevertheless, to address this issue, we examined a more limited set of mutants of a natural /GU-AG/ intron. McConnell et al. (2002) determined the branch sites of a number of U12-dependent introns including the third intron of the Xenopus RPL1 gene (termed the XRP intron). This /GU-AG/ intron has $5^{\prime}$ splice site, $3^{\prime}$ splice site, and branch site sequences that are good matches to the consensuses for these intron elements. It uses a branch site that is located $1 \mathrm{nt}$ upstream in the branch site consensus sequence that is used in the P120 intron (McConnell et al. 2002). The branch site to $3^{\prime}$ splice site distance for this intron is thus $14 \mathrm{nt}$, which is similar to other /GU-AG/ introns.

We constructed two mutant forms of this intron and tested the wild type and both mutants for splicing in vitro (Fig. 6). In the first mutant, XRP oligo CAG, CAG sequences were positioned $8,11,14,17$, and $20 \mathrm{nt}$ from the branch site. In the second mutant, XRP AG $+11+15$, two CAG sequences were positioned 11 and $15 \mathrm{nt}$ from the mapped branch site. These mutants and the wild-type intron were transcribed and spliced in vitro. Figure 6A shows that all the constructs spliced to a similar extent. The spliced exon products were isolated, reverse transcribed, and amplified by PCR as before and were analyzed by polyacrylamide gel electrophoresis (Fig. 6B) and direct sequencing. The results show that the wild-type intron spliced to the normal $+143^{\prime}$ splice site in vitro as expected (Fig. 6C). The oligo CAG construct spliced mainly to the second $A G$ at +11 with some use of the AG at +14 . The double CAG construct used the branch site proximal $+113^{\prime}$ splice site almost exclusively. These results are very similar to the results seen with the /GU-AG/ form of the P120 intron investigated above.

\section{DISCUSSION}

The results described here extend our understanding of the role of several conserved features of U12-dependent intron splice sites. The results largely agree with the constraints on the splice sites that can be inferred from the sequences of suspected U12-type introns derived from database searches (Levine and Durbin 2001; Zhu and Brendel 2003). These analyses suggested that there is some flexibility in the use of splice sites that differ from the majority /AU-AC/ and /GU- 
AG/ patterns. However, possible errors in the underlying sequence data, annotations, and the U12-intron identification algorithms raised uncertainty as to the reality of this apparent flexibility. The results here support this flexibility by investigating various altered splice sites in the context of a well-understood U12-dependent intron.

\section{Roles of the first and last intron nucleotides}

Previous investigations of the role of the first and last nucleotides of U2-dependent introns indicated that these were critical nucleotides in splice site selection and splicing. As discussed above, virtually all known U2-dependent introns throughout eukaryotic phylogeny begin and end with $G$ residues with only a tiny number of exceptions. In contrast, the U12-dependent introns use two different pairs of terminal nucleotides, G-G and A-C, with database evidence suggesting that others can also be used.

In the work reported here, we have tested all possible terminal nucleotide pairs for splicing via the U12dependent spliceosome. The results are in line with the database results and demonstrate that a number of different terminal nucleotide pairs are compatible with correct U12-dependent splicing. In particular, the results show that a $5^{\prime}$ A can splice to any $3^{\prime}$ nucleotide, although a properly positioned AC/ dinucleotide is the preferred site. A $5^{\prime} \mathrm{G}$ residue can splice to $3^{\prime} \mathrm{G}$ or $\mathrm{U}$ residues with a preference for $\mathrm{G}$ residues. Little or no splicing to $3^{\prime} \mathrm{A}$ or $\mathrm{C}$ residues could be detected. This result provides the best evidence that the first and last nucleotides interact in some way since the identity of one of the residues affects the use of the other in an allele-specific manner. The fact that many more combinations appear to be active for U12-dependent splicing suggests that a particular non-Watson-Crick base-pairing arrangement is probably not required. Instead, as was argued previously (Luukkonen and Seraphin 1997), the results appear to be more consistent with an interaction with a common component that binds to both nucleotides.

The greater flexibility of the U12-dependent spliceosome in terminal nucleotide usage compared to the U2dependent spliceosome may reflect underlying differences in the way the splice sites are selected. U1 snRNA base pairs to the $5^{\prime}$ splice site $\mathrm{G}$ residue of U2-type introns. This $\mathrm{G}$ residue provides a considerable amount of the information content of U2-type $5^{\prime}$ splice sites (Burge et al. 1999) and the U1 base pair to this residue provides a significant amount of the energy in this interaction. In contrast, the $5^{\prime}$ terminal residue of the U12-dependent splice site does not appear to base pair to U11 or U6atac snRNAs and provides a much smaller fraction of the information content of U12-type splice sites (Burge et al. 1999). Therefore, at the level of splice site selection, the $5^{\prime}$ residue may play a more important role in U2- as opposed to U12-type introns.

At the $3^{\prime}$ splice site, the terminal $\mathrm{G}$ residue in U2-type introns appears to interact with the small subunit of U2AF
(Merendino et al. 1999; Wu et al. 1999; Zorio and Blumenthal 1999). This interaction has been shown to be sensitive to changes in the identity of the $3^{\prime}$ terminal residue. In the case of U12-dependent introns, a role for the small subunit of $\mathrm{U} 2 \mathrm{AF}$ has not been established. The absence of a polypyrimidine tract in U12-type introns suggests that there is no requirement for binding of the large subunit of $\mathrm{U} 2 \mathrm{AF}$ in this system. Therefore, the $3^{\prime}$ terminal residue may be primarily involved in other interactions such as with Prp8 protein (Collins and Guthrie 1999).

\section{Roles of the +2 and -2 residues in the splice site dinucleotides}

When we examined the requirements for the +2 and -2 residues in U12-dependent splicing, we obtained results that were similar to the U2-dependent splicing system. We found that both the $U$ and $A$ residues were required for efficient splicing. The only exception was some activity in the $3^{\prime}-2 \mathrm{G}$ mutant. Significantly, no combinations of mutants were more active than the individual mutants, suggesting that these residues do not base pair or functionally interact as seen in the first and last nucleotide mutants discussed above. Most splicing events adhered to a /NU to $\mathrm{RN} /$ rule such that the $5^{\prime}$ splice site was always one nucleotide $5^{\prime}$ of a $\mathrm{U}$ residue and the $3^{\prime}$ splice site was always one nucleotide $3^{\prime}$ of an A or G residue. These penultimate residues are the only ones observed to function in vivo as well. Three examples of /GU-GG/ introns were detected by Levine and Durbin (2001), confirming the activity of the $3^{\prime}-2 \mathrm{G}$ mutant in our study (the possibility of /AU-GC/ introns was not investigated by these authors; Levine and Durbin 2001).

One notable difference between the U2- and U12dependent splicing systems revealed by these experiments is in the function of a $5^{\prime}+2 \mathrm{C}$ residue. A $\mathrm{C}$ residue at the +2 position is the only common exception to the /GU-AG/ rule seen in U2-dependent human introns (Clark and Thanaraj 2002). However, no examples of /GC-AG/ or /AC-AC/ U12dependent introns have been found in database searches (Levine and Durbin 2001). In agreement with this, when we tested a +2 C $5^{\prime}$ splice site mutant in vivo, it showed no U12dependent splicing. This result suggests that there may be differences in the recognition of this position in the two splicing systems. However, the mechanistic cause of this difference is unknown.

In spite of the clear preference for the canonical residues at the $5^{\prime}+2$ and $3^{\prime}-2$ positions, it is noteworthy that nonconsensus $3^{\prime}$ dinucleotides can become active when the $3^{\prime}$ splice site is moved to more distal positions as shown in Figure 3. These mutants show splicing of a $5^{\prime} / \mathrm{GU}$ to $3^{\prime} \mathrm{UG} /$ and CG/ dinucleotides. A similar activation of a $5^{\prime} / \mathrm{AU}$ to $3^{\prime} \mathrm{UU} /$ splice was seen in our previous study (Dietrich et al. 2001). Thus, it appears that no single residue is completely indispensable for splicing. Rather, the precise sites of splic- 
ing and the overall activity of splice sites are determined by both the identities of individual nucleotides and the larger sequence context. This flexibility in $3^{\prime}$ splice site usage also appears to occur in natural genes. Levine and Durbin (2001) described 15 cases in which EST evidence supported the alternative use of nonconsensus $3^{\prime}$ splice sites within a few nucleotides of the consensus sites. Many of these splice variants altered the translational reading frame of the downstream exon and are likely to be errors rather than true alternatively splicing isoforms. These observations coupled with our mutant data suggest that the greater flexibility in U12-dependent $3^{\prime}$ splice sites leads to an increased level of splicing errors compared to U2-dependent introns.

\section{Constraints on branch site to $3^{\prime}$ splice site distance in a /GU-AG/ intron}

We showed previously that the $3^{\prime}$ splice of a U12-dependent /AU-AC/ intron had to be located between 10 and $\sim 20$ nt $3^{\prime}$ of the branch site for optimum activity (Dietrich et al. 2001). This spacing constraint agreed with the observed branch site to $3^{\prime}$ splice site distances observed in vivo. We also noted that larger distances were frequently observed in the /GU-AG/ subclass of U12-dependent introns (Dietrich et al. 2001), a finding borne out in a larger sample (Levine and Durbin 2001). Here we investigated this spacing constraint further by creating a /GU-AG/ intron with different spacings between the branch site and the $3^{\prime}$ splice site. We found that smaller distances were active using /GU-AG/ termini than were found previously using /AU-AC/ termini. Spacings of 8 and 9 were fully active in the present case with /GU-AG/ termini whereas these distances led to cryptic site activation in our previous experiments with /AU-AC/ termini (Dietrich et al. 2001). This agrees with the results of Levine and Durbin (2001) where several introns with spacings of 8 and 9 were predicted. Significantly, no introns were found with predicted distances of $<8 \mathrm{nt}$.

In database searches, the optimum distance appears to be slightly different in the two subclasses. The predicted distances for the /AU-AC/ class show a sharp peak at $12 \mathrm{nt}$ with $85 \%$ of introns falling in the range of $10-14 \mathrm{nt}$. The /GU-AG/ class has a broader peak between 12 and $16 \mathrm{nt}$ with $80 \%$ of introns falling in this range (Dietrich et al. 2001; Levine and Durbin 2001). In our experimental system, the /AU-AC/ intron showed a very pronounced optimum at $12 \mathrm{nt}$ in a construct with potential sites at $8,10,12$ and $14 \mathrm{nt}$ (Dietrich et al. 2001). Here, in a similar P120 derived /GU-AG/ construct with potential sites at 7, 10, 13, and $16 \mathrm{nt}$, both the 10 and 13 sites were used, although the site at +10 was used preferentially. In addition, when a different construct with potential sites at 8 and 12 was tested, both sites were used roughly equally. Furthermore, using an authentic /GU-AG/ intron (XRP; Fig. 6) where the wild-type branch site is known (McConnell et al. 2002), splicing occurs preferentially at a spacing of $11 \mathrm{nt}$.
These experimental results suggest that a spacing of 10$11 \mathrm{nt}$ is optimum for /GU-AG/ introns, while $12 \mathrm{nt}$ is optimum for /AU-AC/ introns. While there is good agreement between the database and experimental optima for the /AU-AC/ class, there is a discrepancy in the optima for the /GU-AG/ class. This may be a reflection of the greater specificity of a /GU $5^{\prime}$ splice site to splice to a $3^{\prime} \mathrm{G} /$ residue compared with the ability of a /AU $5^{\prime}$ splice site to utilize any $3^{\prime}$ nucleotide. Therefore, there would be fewer potential sites of missplicing in the $3^{\prime}$ splice site region of a /GU-AG/ intron, allowing for a more relaxed distance constraint such as we observe in the databases.

The maximum allowable distance appears to be similar in both types of U12-type introns (Dietrich et al. 2001; present study). Here, when the $3^{\prime}$ splice site AG/ was moved to +18 , we observed the activation of cryptic nonconsensus splice sites at the +12 and +13 positions, although most splicing occurred at the +18 site. Moving the $3^{\prime}$ splice site to more distal positions further activated the cryptic sites especially in the +37 mutant where the cryptic sites were used preferentially. These results confirm that there is a strong position dependence in the choice of U12-dependent $3^{\prime}$ splice sites.

A recent analysis of U12-dependent introns in the plant Arabidopsis thaliana (Zhu and Brendel 2003) concluded that the functional branch site to $3^{\prime}$ splice site distance in this organism was greater than we previously suggested (Dietrich et al. 2001). Putting aside the possible differences in splicing systems due to the large phylogenetic distance between plants and vertebrates, we would simply point out that increasing distances in our experiments are associated with greater activation of nonconsensus cryptic $3^{\prime}$ splice sites closer to the branch point. If such cryptic sites were highly selected against in introns with large distances, the consensus $3^{\prime}$ splice site could still be functional. In fact, the Zhu and Brendel (2003) study demonstrated biased representation of several dinucleotides in the $3^{\prime}$ splice site region in their database. Furthermore, recent searches for U12dependent introns in the human genome have revealed a few cases of branch to $3^{\prime}$ splice site distances longer than 30 nt (R. Padgett and C. Burge, unpubl.). Nevertheless, the data shown here and in our previous study (Dietrich et al. 2001) indicate that increasing distances are associated with decreased fidelity in splice site usage that will place a limit on the functional distance in U12-dependent introns.

\section{Application of these results to a human disease}

While this article was in preparation, an interesting example of some of the points made here appeared. Hastings et al. (2005) report on a mutation in one of the splice sites of the human LKB1 gene, a tumor suppressor gene whose germline inactivation leads to Peutz-Jeghers syndrome. As noted by these authors, the second intron of this gene is a U12-dependent intron of the /AU-AC/ subclass. The muta- 
tion they describe converts the first nucleotide of the intron to a $G$ residue. This mutation leads to the missplicing of the intron and the production of nonfunctional mRNAs. As shown in our data above and previously (Dietrich et al. 1997) this A to $G$ change will prevent splicing to the normal $3^{\prime}$ splice site $C$ residue and will likely lead to activation of nearby cryptic $3^{\prime}$ splice sites with terminal G or $U$ residues. This was, in fact, what was observed. Of 19 cDNA clones from the mutant allele, 9 spliced to a CG $/ 3^{\prime}$ splice site dinucleotide at $+1,5$ spliced to an $\mathrm{AU} /$ at +4 , 4 spliced to a UG/ at +7 , and 1 spliced to a GG/ at +8 (Hastings et al. 2005). The normal branch site to $3^{\prime}$ splice site spacing in this intron is $10 \mathrm{nt}$, which places the cryptic $3^{\prime}$ splice sites at positions 11-18. This is well within the preferred distance range that we determined here and previously (Dietrich et al. 2001). These results support the idea that the patterns in U12-dependent splice site selection that we have described here and in previous work have applicability in the understanding of the expression of genes in health and disease.

\section{MATERIALS AND METHODS}

\section{Construction of mutant DNA constructs}

The construction of the P120 intron F minigene and mutants derived from it are as described (Kolossova and Padgett 1997). The mutants were produced by PCR mutagenesis using oligonucleotides with the desired mutations as described previously (Dietrich et al. 2001). The XRP intron mutants were derived from the construct described by McConnell et al. (2002), kindly provided by J. Steitz (Yale University). All mutations were confirmed by DNA sequencing.

\section{Analysis of in vivo splicing}

Transient transfection of the P120 minigene expression plasmid into cultured CHO cells was as described (Hall and Padgett 1996; Kolossova and Padgett 1997). For these experiments, $1 \mu \mathrm{g}$ of P120 plasmid and $9 \mu \mathrm{g}$ of pUC19 carrier DNA were added to $1 \times 10^{6}$ cells. Total RNA was isolated from cells $48 \mathrm{~h}$ after transfection, reverse transcribed using a vector specific primer, and amplified by PCR using P120 exon 6 and 7 specific primers as described (Kolossova and Padgett 1997; Dietrich et al. 2001). For highresolution gels, the exon 7 primer was $5^{\prime}$-end labeled with ${ }^{32} \mathrm{P}$ using T4 polynucleotide kinase and $\gamma_{-}{ }^{32} \mathrm{P}$ ATP. The products were analyzed by agarose gel electrophoresis, visualized using ethidium bromide, and photographed using a digital video camera (BioPhotonics). Single nucleotide resolution of labeled PCR products was obtained by electrophoresis in $8 \%$ polyacrylamide gels containing $7 \mathrm{M}$ urea and $40 \%$ formamide. Radioactivity was detected using a Storm Imager (Molecular Dynamics). To verify the sites of splicing used in the various mutants, PCR products were separated on agarose or nondenaturing polyacrylamide gels. Bands were excised, reamplified, purified by gel electrophoresis, and sequenced. Independent transfections and analyses gave substantially similar results.

\section{In vitro splicing reactions}

DNA templates for in vitro transcription were prepared by PCR amplification using the P120 minigene constructs with $3^{\prime}$ splice site sequences shown in Figure 3 as described (Dietrich et al. 1997, 2001). For the XRP wild-type and mutant templates, a common $5^{\prime}$ PCR primer located in the upstream exon (GCGAAGCTTAATACGACT CACTATAG) was used with specific $3^{\prime}$ primers that included the branch site, $3^{\prime}$ splice site(s), and downstream exon (XRP wild type: GGAGTACTTACCCCAACAGAGCGGATAACAATTTCACACAGG CTTCTGTGTTGTATTGAC; XRP oligo CAG: CTGTGTTGTATT GACTCTACGATGCCAGCGTCTCCAGGTCTTAGTTGGGGCAAA CATACGCCCACCACGCTGCTGCTGCTGCTGATATTAAGGAAA ATA; XRP AG+11+15: CTGTGTTGTATTGACTCTACGATGC CAGCGTCTCCAGGTCTTAGTTGGGGCAAACATACGCCCACCA CGACACATCTGTCTGCAAATATTAAGGAAAATA). In all cases, a consensus U2-dependent $5^{\prime}$ splice site was located at the $3^{\prime}$ end of the transcripts to promote efficient U12-dependent splicing ( $\mathrm{Wu}$ and Krainer 1996). RNA was synthesized from these templates using T7 RNA polymerase, gel purified, and equal amounts of each RNA were spliced in vitro for $3 \mathrm{~h}$ in the presence of an antisense $2^{\prime}$-O-methyl oligonucleotide directed against U2 snRNA as described (Dietrich et al. 1997, 2001). An antisense 2'-O-methyl oligonucleotide against U12 snRNA was included where indicated. RNA from splicing reactions was separated on $8 \%$ polyacrylamide gels under denaturing conditions and detected with a Molecular Dynamics Storm Imager. For RT-PCR analysis of in vitro splicing reactions, spliced RNAs from preparative scale reactions were separated on gels as above and RNA extracted from the spliced exons region. The RNA was reversed transcribed with a $3^{\prime}$ end-specific primer using Superscript reverse transcriptase and PCR amplified using exon specific primers.

\section{ACKNOWLEDGMENTS}

We thank Robert Incorvaia and Andrew Seyboldt for construction of some of the mutants used in this study, and J. Steitz for the XRP construct. This work was supported by grant GM55105 from the National Institutes of Health.

Received October 14, 2004; accepted May 19, 2005.

\section{REFERENCES}

Aebi, M., Hornig, H., Padgett, R.A., Reiser, J., and Weissmann, C. 1986. Sequence requirements for splicing of higher eukaryotic nuclear pre-mRNA. Cell 47: 555-565.

Burge, C.B. and Sharp, P.A. 1997. Classification of introns: U2-type or U12-type. Cell 91: 875-879.

Burge, C.B., Tuschl, T., and Sharp, P.A. 1999. Splicing of precursors to mRNAs by the spliceosome. In The RNA world, 2d ed. (eds. R.F. Gestland et al.), pp. 525-560. Cold Spring Harbor Laboratory Press, Cold Spring Harbor, NY.

Chanfreau, G. and Jacquier, A. 1993. Interactions of intronic boundaries is required for the second splicing step efficiency of a group II intron. EMBO J. 12: 5173-5180.

Chanfreau, G., Legrain, P., Dujon, B., and Jacquier, A. 1994. Interaction between the first and last nucleotides of pre-mRNA introns is a determinant of $3^{\prime}$ splice site selection in S. cerevisiae. Nucleic Acids Res. 22: 1981-1987. 
Clark, F. and Thanaraj, T.A. 2002. Categorization and characterization of transcript-confirmed constitutively and alternatively spliced introns and exons from human. Hum. Mol. Genet. 11: 451-464.

Collins, C.A. and Guthrie, C. 1999. Allele-specific genetic interactions between Prp8 and RNA active site residues suggest a function for Prp8 at the catalytic core of the spliceosome. Genes \& Dev. 13: 1970-1982.

Crick, F. 1979. Split genes and RNA splicing. Science 204: 264-271.

Deirdre, A., Scadden, J., and Smith, C.W. 1995. Interactions between the terminal bases of mammalian introns are retained in inosinecontaining pre-mRNAs. EMBO J. 14: 3236-3246.

Dietrich, R.C., Incorvaia, R., and Padgett, R.A. 1997. Terminal intron dinucleotide sequences do not distinguish between U2- and U12dependent introns. Mol. Cell 1: 151-160.

Dietrich, R.C., Peris, M.J., Seyboldt, A.S., and Padgett, R.A. 2001. Role of the $3^{\prime}$ splice site in U12-dependent intron splicing. Mol. Cell. Biol. 21: 1942-1952.

Gaur, R.K., Beigelman, L., Haeberli, P., and Maniatis, T. 2000. Role of adenine functional groups in the recognition of the $3^{\prime}$-splice-site AG during the second step of pre-mRNA splicing. Proc. Natl. Acad. Sci. 97: 115-120.

Hall, S.L. and Padgett, R.A. 1994. Conserved sequences in a class of rare eukaryotic introns with non-consensus splice sites. J. Mol. Biol. 239: 357-365.

- 1996. Requirement of U12 snRNA for the in vivo splicing of a minor class of eukaryotic nuclear pre-mRNA introns. Science 271: 1716-1718.

Hastings, M.L., Resta, N., Traum, D., Stella, A., Guanti, G., and Krainer, A.R. 2005. An LKB1 AT-AC intron mutation causes Peutz-Jeghers syndrome via splicing at noncanonical cryptic splice sites. Nat. Struct. Mol. Biol. 12: 54-59.

Incorvaia, R. and Padgett, R.A. 1998. Base pairing with U6atac snRNA is required for $5^{\prime}$ splice site activation of U12-dependent introns in vivo. RNA 4: 709-718.

Kolossova, I. and Padgett, R.A. 1997. U11 snRNA interacts in vivo with the 5 ' splice site of U12-dependent (AU-AC) introns. RNA 3: 227-233.

Levine, A. and Durbin, R. 2001. A computational scan for U12dependent introns in the human genome sequence. Nucleic Acids Res. 29: 4006-4013.
Luukkonen, B.G.M. and Seraphin, B. 1997. The role of branchpoint- $3^{\prime}$ splice site spacing and interaction between intron terminal nucleotides in $3^{\prime}$ splice site selection in Saccharomyces cerevisiae. EMBO J. 16: 779-792.

McConnell, T.S., Cho, S.J., Frilander, M.J., and Steitz, J.A. 2002. Branchpoint selection in the splicing of U12-dependent introns in vitro. RNA 8: 579-586.

Merendino, L., Guth, S., Bilbao, D., Martinez, C., and Valcarcel, J. 1999. Inhibition of msl-2 splicing by sex-lethal reveals interaction between U2AF35 and the $3^{\prime}$ splice site AG. Nature 402: 838-841.

Parker, R. and Siliciano, P.G. 1993. Evidence for an essential nonWatson-Crick interaction between the first and last nucleotides of a nuclear pre-mRNA intron. Nature 361: 660-662.

Reed, R. and Maniatis, T. 1985. Intron sequences involved in lariat formation during pre-mRNA splicing. Cell 41: 95-105.

Ruis, B.L., Kivens, W.J., and Siliciano, P.G. 1994. The interaction between the first and last intron nucleotides in the second step of pre-mRNA splicing is independent of other conserved intron nucleotides. Nucleic Acids Res. 22: 5190-5195.

Tarn, W.Y. 1996. Site-specific substitution of inosine at the terminal positions of a pre-mRNA intron: Implications for the configuration of the terminal base interaction. Biochimie 78: 1057-1065.

Tarn, W.-Y. and Steitz, J.A. 1996. A novel spliceosome containing U11, U12 and U5 snRNPs excises a minor class (AT-AC) intron in vitro. Cell 84: 801-811.

Wu, Q. and Krainer, A.R. 1996. U1-mediated exon definition interactions between AT-AC and GT-AG introns. Science 274: 10051008.

1997. Splicing of a divergent subclass of AT-AC introns requires the major spliceosomal snRNAs. RNA 3: 586-601.

Wu, S., Romfo, C.M., Nilsen, T.W., and Green, M.R. 1999. Functional recognition of the $3^{\prime}$ splice site AG by the splicing factor U2AF35. Nature 402: 832-835.

Zhu, W. and Brendel, V. 2003. Identification, characterization and molecular phylogeny of U12-dependent introns in the Arabidopsis thaliana genome. Nucleic Acids Res. 31: 4561-4572.

Zorio, D.A.R. and Blumenthal, T. 1999. Both subunits of U2AF recognize the $3^{\prime}$ splice site in Caenorhabditis elegans. Nature 402: 835-838. 

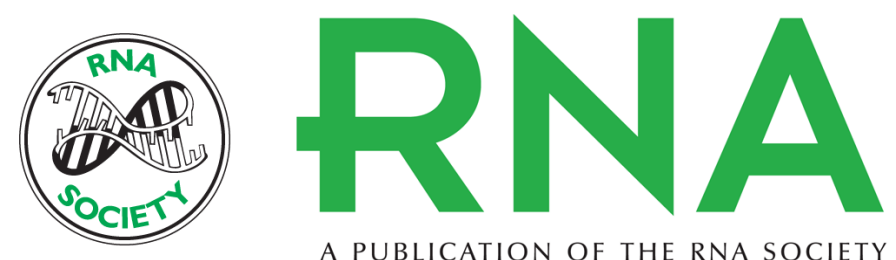

A PUBLICATION OF THE RNA SOCIETY

\title{
A mutational analysis of U12-dependent splice site dinucleotides
}

\author{
ROSEMARY C. DIETRICH, JOHN D. FULLER and RICHARD A. PADGETT
}

RNA 2005 11: 1430-1440

References This article cites 30 articles, 11 of which can be accessed free at:

http://rnajournal.cshlp.org/content/11/9/1430.full.html\#ref-list-1

License

Email Alerting Receive free email alerts when new articles cite this article - sign up in the box at the Service top right corner of the article or click here.

To subscribe to $R N A$ go to:

http://rnajournal.cshlp.org/subscriptions 